\title{
PCR MULTIPLEX IN THE DETECTION OF Tinea unguium IN PATIENTS FROM CARACAS - VENEZUELA.
}

\begin{abstract}
Alvarado Primavera1 ${ }^{1}$, Camacaro Laurymar², De Arbeloa Michelle², Fernández Alexis ${ }^{3}$, Cavallera Elsy². Laboratorio de Micología Médica1', Especialización en Dermatología y Sifilografía². Laboratorio de Inmunología II ${ }^{3}$. Servicio Autónomo Instituto de Biomedicina Dr. Jacinto Convit. Caracas, Venezuela.
\end{abstract}

Background: Tinea unguium is a superficial mycosis produced by dermatophytes that infect the nail plate of hands and feet. The superficial mycoses are cosmopolitan and for their high frequency, they are a serious public health problem. The conventional diagnosis is based on the observation of the clinical samples and the mycological culture for the morphological identification of the fungus. The development of molecular biology techniques such as PCR multiplex (PCRm), it allows a better identification and diagnosis of the causative agents of $T$. unguium. Aims: To evaluate and standardize the PCRm technique in the diagnosis of dermatophytes in the nails. Methods: 49 patients were evaluated with the diagnostic impression of Tinea unguium and 10 samples of healthy individuals. Scraping of the nail bed was performed for direct examination and mycological culture. For molecular analysis distal section of nail plate was made, obtaining 6-36 mg of sample. The extraction of the total DNA from the nail samples was carried out with the Wizard $\AA$ Genomic DNA Purification Kit (Promega). Specific primers were used for the ITS regions of $\mathrm{T}$. rubrum (5'CCC CCC ACG ATA GGG ACCG'3 and 5 'GAC TGA CAG CTC TTC AGA GAA TT 3'), T. mentagrophytes (5 'GCC CCC CAC GAT AGG GCC AA 3 'and 5' CTC GCC GAA CGG CTC TCC TG 3 '). Specific sequences of the chitin synthetase I (QSI) gene present in all dermatophyte species were used (5 'GAA GCC TGG AAG AAG ATT GTC G 3' and 5 'CCT TGA TTT CAC CGC AGG CAC 3'). DNA amplification was performed in an Icycler thermocycler (BioRad), with a first denaturation cycle at $95^{\circ} \mathrm{C}$ for $5 \mathrm{~min}$. The amplification program for the PCRm consisted of 30 cycles of $94^{\circ} \mathrm{C}$ for $30 \mathrm{~s}$, followed by $62^{\circ} \mathrm{C}$ for 30 $\mathrm{s}$ and finally $72{ }^{\circ} \mathrm{C}$ for $30 \mathrm{~s}$. Finishing with an extension of $72^{\circ} \mathrm{C}$ during $10 \mathrm{~min}$. The mixtures were resolved on $2 \%$ agarose gels at $60 \mathrm{~V}$ and stained with ethidium bromide.

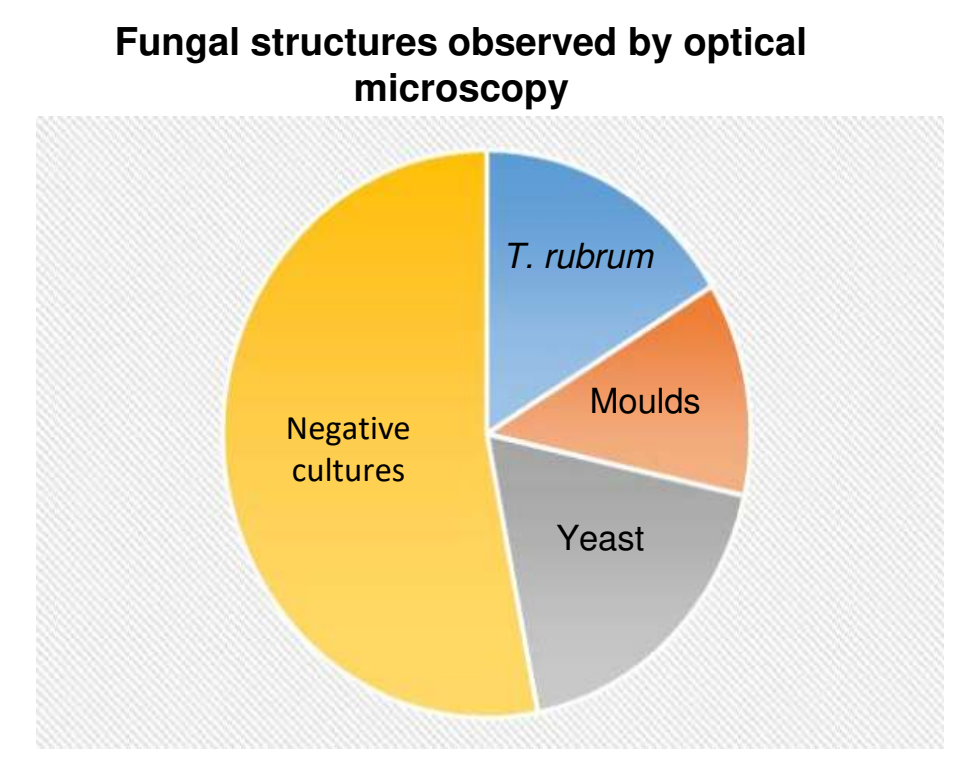

Results: The patients studied, $63.3 \%$ corresponded to the female gender. By optical microscopy, $28.6 \%$ yeasts and $55.1 \%$ hyphae were observed. of the samples processed, 8 cultures of T. rubrum (16.3\%), 2 moulds (4.0\%) and 9 yeasts (18.4\%) were isolated. Regarding PCRm, $132 \mathrm{bp}$ amplification products were observed corresponding to $T$. mentagrophytes (lines 5-8), 214 bp for T. rubrum (lines 1-4) and 432 bp for other dermatophytes (lines 9, 10) (figure 1). The PCRm was positive for dermatophytes in 19 patients (38.8\%), overcoming the mycological culture by 11 samples, of which 13 corresponded to T. rubrum (26.5\%), 4 to T. mentagrophytes (8.2\%) and 2 other dermatophytes (4.1\%). a sensitivity of $97 \%$ was obtained, a specificity of $73.2 \%$. Conclusion: These results agree with those reported in the literature, finding that the sensitivity and specificity, of the PCRm ranges between 85 and $97 \%$.

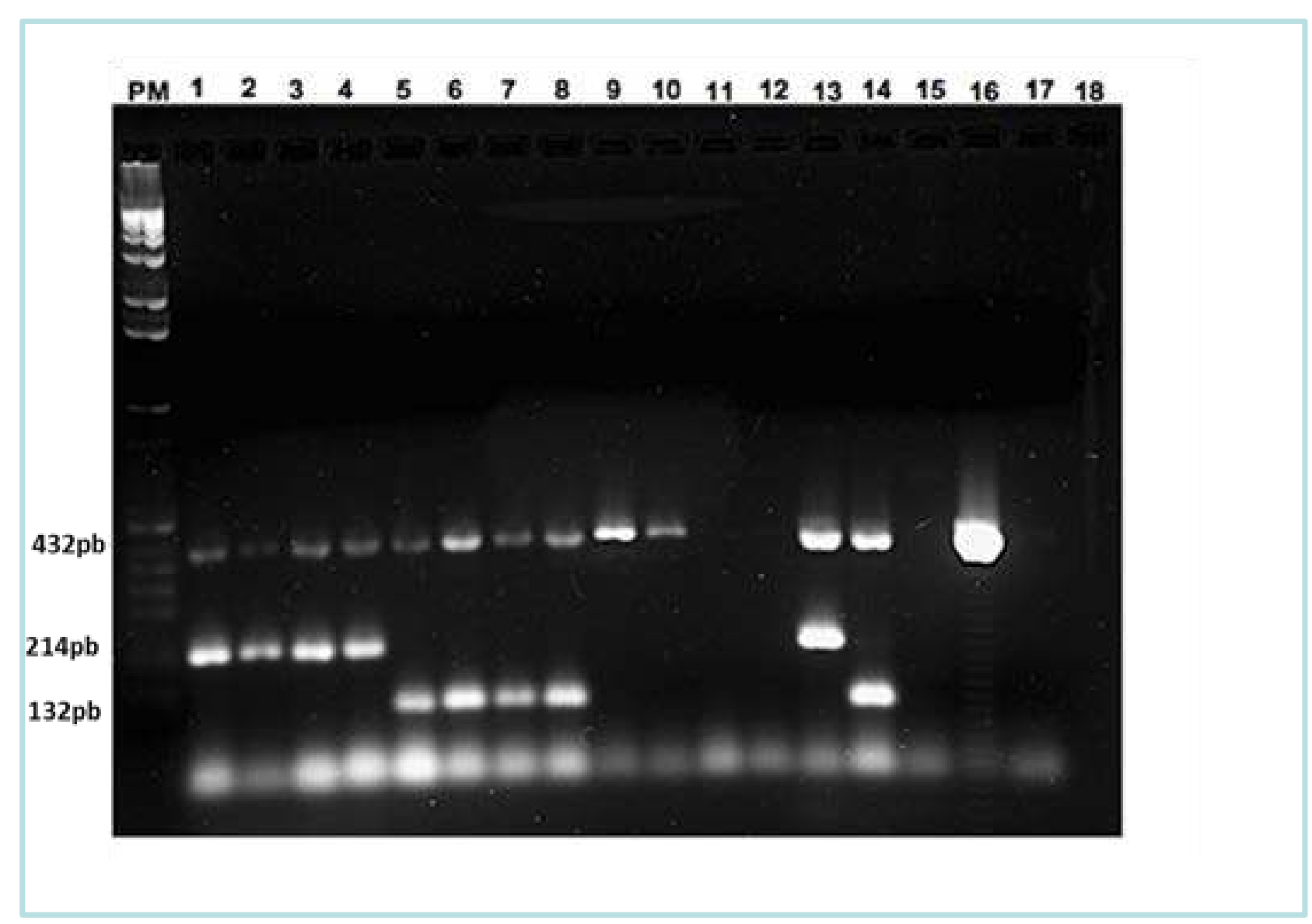

PCRm for detection of dermatophytes: Amplification products resolved in $2 \%$ agarose gel (PM): Molecular weights. (1-4): Positive patients for T rubrum. (5-8): Positive patients for T mentagrophytes. $(9,10)$ : Positive patients for Dermartophytes. $(11,12)$ : Healthy Controls. (13): Positive control T. rubrum. (14): Positive control T. mentagrophytes. (15): Negative control Candida albicans. (16): Positive control T. tonsurans. (17): Negative control without DNA 